\title{
Development of a sustained-release microcapsule for delivery of metoprolol succinate
}

\author{
LI SONG $^{1}$, SHENGJIANG HE ${ }^{2}$ and QINENG PING ${ }^{1}$ \\ ${ }^{1}$ Department of Pharmaceutics, School of Pharmacy, China Pharmaceutical University, Nanjing, Jiangsu 210009; \\ ${ }^{2}$ Department of Traditional Chinese Medicine, Guangdong Research Institute, Guangzhou, Guangdong 510000, P.R. China
}

Received September 10, 2015; Accepted October 11, 2016

DOI: $10.3892 / \mathrm{etm} .2017 .4247$

\begin{abstract}
Sustained-release (SR) formulations of metoprolol succinate (MS) may minimize fluctuations in plasma concentration and decrease the resulting adverse events. The aim of the present study was to optimize the loading capacity of microcapsules and the SR of MS. A uniform design method was applied to optimize the formulation of SR microcapsules, composed of ethyl cellulose and polyethylene glycol 6,000, in one step via emulsion-solvent diffusion. In vitro release was studied, and the in vivo bioavailability of MS following dosing with novel microcapsules was compared with a commercially available MS formulation in beagle dogs. The present methodology achieved an entrapment efficiency of $83.2 \%$, with $96.1 \%$ of drug released in vitro in $18 \mathrm{~h}$, and the release was close to linear over a 12 -h period. Pharmacokinetic studies of MS microcapsules in beagle dogs demonstrated a superior SR profile compared with conventional SR tablets. MS microcapsules were developed with high encapsulation efficiency, which had desirable SR properties in vitro and in vivo.
\end{abstract}

\section{Introduction}

Metoprolol succinate (MS) is a highly selective $\beta_{1}$-adrenergic receptor blocker typically used for the treatment of hypertension, coronary heart disease, chronic heart failure and arrhythmia (1). However, at higher plasma concentrations, MS may also inhibit $\beta_{2}$-adrenergic receptors located in the vascular and bronchial musculature. Additionally, when plasma MS concentrations are greater than required, anesthetic membrane-stabilizing activity is detected $(2,3)$. Therefore, it is necessary to control the plasma MS concentration to maximize therapeutic effects and minimize side effects. Due to the short half-life of metoprolol (3-4 h) MS must be administered

Correspondence to: Dr Qineng Ping, Department of Pharmaceutics, School of Pharmacy, China Pharmaceutical University, 24 Tong Jia Xiang, Nanjing, Jiangsu 210009, P.R. China E-mail: pingqn0723@sina.com

Key words: metoprolol succinate, sustained-release, microencapsulation, pharmacokinetics, entrapment efficiency several times a day, leading to fluctuations in plasma MS concentration (4). Sustained-release (SR) formulations are able to minimize fluctuations in plasma concentration, hence minimizing the adverse effects associated with excessively high plasma concentrations and providing an effective stable dose (5). This may be achieved through various formulations, including hydrophilic matrix tablets, SR pellets, osmotic pump tablets and drug-polymer conjugates. Novel platforms, such as nano/microcarriers with SR, improved adhesion and tissue penetration, may be utilized for oral drug delivery (6).

Microcapsules provide several advantages compared with conventional dosage forms, including modulated drug release, enhanced drug stability and reduced gastrointestinal irritation (7). Microspheres have been previously used in an SR formulation of MS to provide robust and consistent control of hypertension and heart rate (8). Recently, metoprolol tartrate sustained-release capsules, which are polymer-coated metoprolol tartrate matrix granules, have become commercially available. Hydrophilic hydroxypropyl methyl cellulose (HPMC) and hydrophobic ethyl cellulose polymers may also be employed as matrix builders, and Eudragit ${ }^{\circledR}$ RL/RS as a coating polymer. Microparticles coated with a film composed of these water-insoluble polymers exhibit extended release periods of up to $12 \mathrm{~h}$ in vitro (9).

Various encapsulation methods may improve immobilization, isolation and protection of commercial products and facilitate control of the transfer rate of pharmacological agents, nutrients and perfumes (10). The emulsification-solvent diffusion method is an established method for the preparation of microcapsules based on an emulsion technique. This method involves adding capsule material dissolved in an organic solution to an aqueous solution saturated with organic solvent to form an emulsion. Removal of organic solvents induces the formation of microcapsules as a result of phase separation due to the decreased solubility of the capsule material in solution. These formulations produce microcapsules with an evenly rounded shape, a smooth surface and good SR characteristics $(11,12)$. Ethylcellulose (EC) is a non-ionic, pH-insensitive cellulose ether, which is insoluble in water but soluble in numerous polar organic solvents (13), and exhibits SR properties. Dash et al (14) previously confirmed that aspirin-loaded EC microcapsules, made using the emulsion solvent evaporation method, were able to effectively reduce the drug release rate. Polyethylene glycol (PEG) is a hydrophilic polymer 
material and plasticizer. PEG enhances the flexibility and plasticity of microcapsules, decreases the tendency for aggregation and adhesion, and improves dispersion. It may also be used as a porogen for membrane-controlled drug release, and, with a suitable ratio of polymer semi-permeable membrane and porogenic materials, is able to reduce the rate of drug release (15).

The objective of the present study was to develop a method of MS encapsulation to provide microcapsules with high entrapment efficiency and optimal SR profiles in vitro and in vivo. The emulsification-solvent diffusion method (14) was used to prepare microcapsules of ethyl cellulose and PEG 6,000 and the release of MS from these capsules was assessed in vitro and in vivo.

\section{Materials and methods}

Preparation of SR microcapsules of MS. MS (AstraZeneca Pharmaceuticals Co., Ltd., Wuxi China) was dissolved in water (the internal aqueous phase, $\mathrm{W}_{1}$ ) and added to $2 \mathrm{ml}$ ethyl acetate (EA) solution (O) and ultrasonically emulsified, forming a primary emulsion $\left(\mathrm{W}_{1} / \mathrm{O}\right)$. The indicated concentration of PEG 6,000 (Sigma-Aldrich; Merck Millipore, Darmstadt, Germany) was dissolved in EA-saturated water (containing 0.5\% SDS; Tianjin Global Chemical Technology Co., Ltd., Tianjin, China) to constitute the external aqueous phase $\left(\mathrm{W}_{2}\right)$. $\mathrm{W}_{1} / \mathrm{O}$ was slowly added drop-wise to $\mathrm{W}_{2}$, under continuous stirring until the emulsion droplets solidified to form a stable $\mathrm{W}_{1} / \mathrm{O} / \mathrm{W}_{2}$ complex emulsion. Water was used to dilute the emulsion 20 -fold. The ethyl cellulose phase separated and condensed into a capsule, and following sedimentation of the microcapsules, the supernatant was removed and the retentate centrifuged and filtered to obtain the microcapsules, which were subsequently washed with water and dried in a vacuum at $40^{\circ} \mathrm{C}$.

Study of microcapsule morphology and size distribution. Microcapsules were re-suspended in double-distilled water via ultrasonic dispersion for $20 \mathrm{~min}$. The sample was then observed under a microscope (Olympus X 51, Olympus Corporation, Tokyo, Japan; magnification, x400) and particle diameter was measured.

Determination of MS drug loading capacity and encapsulation efficiency. The maximum absorption of MS was previously recorded at $274 \mathrm{~nm}$ when scanned at a wavelength of 200-350 nm (16). Therefore, $274 \mathrm{~nm}$ was chosen as the optimum wavelength for measuring the MS content. A total of $50 \mathrm{mg}$ of microcapsules were placed in a $100-\mathrm{ml}$ volumetric flask and dissolved in ethanol. The solution was ultrasonically treated for $30 \mathrm{~min}$ and filtered through a $0.8-\mu \mathrm{m}$ microporous membrane filter. Of the resulting filtrate, $5 \mathrm{ml}$ was diluted with $20 \mathrm{ml}$ water and the ultraviolet (UV) absorbance at $274 \mathrm{~nm}$ was measured using an ultraviolet spectrophotometer (Shimadzu UV2550; Shimadzu Corporation, Kyoto, Japan). MS drug loading capacity was calculated as: Quantity of agent in microcapsules/microcapsule weight $\mathrm{x} 100$. Encapsulation efficiency was calculated as follows: Quantity of agent in the microcapsules/total quantity of agent used x100.
A linear correlation was obtained with $\mathrm{A}=0.0042 \mathrm{C}+0.0014$ $(r=0.9997)$, Where A is the absorbance intensity, $\mathrm{C}$ is the concentration of drug and $\mathrm{r}$ is the linearly dependent coefficient. The intra-day relative standard deviation (RSD) was $0.41 \%(n=6)$, and the inter-day RSD was $0.85 \%(n=6)$, with the average recovery being 99.2 and the average RSD being $0.37 \%(\mathrm{n}=9)$.

MS microcapsule in vitro release. Artificial gastric fluid was prepared by diluting $16.4 \mathrm{ml}$ hydrochloric acid in $800 \mathrm{ml}$ water and $10 \mathrm{~g}$ pepsin $(3,800 \mathrm{U} / \mathrm{mg}$; Sichuan Deyang Biochemical Products Co., Ltd., Deyong, China) and diluting again with water to $1,000 \mathrm{ml}$. Artificial intestinal fluid was prepared by dissolving $6.8 \mathrm{~g}$ potassium dihydrogen phosphate in $500 \mathrm{ml}$ water, which was adjusted to $\mathrm{pH} 6.8$ with sodium hydroxide, to which $10 \mathrm{~g}$ trypsin (2,500 U/mg; Sichuan Deyang Biochemical Products Co., Ltd.) was added, followed by further dilution with water to $1,000 \mathrm{ml}$.

Prepared microcapsules were placed in a basket with $1,000 \mathrm{ml}$ of release medium (water, artificial gastric fluid or artificial intestinal fluid) and the rotation speed was fixed at $100 \mathrm{rpm}$ at $37 \pm 0.5^{\circ} \mathrm{C}$. A $5-\mathrm{ml}$ sample was taken from the release solution at predetermined time intervals $(0.5,2,4,6$, $8,10,12,15$ and $18 \mathrm{~h}$ ) and $5 \mathrm{ml}$ of release medium (water, artificial gastric fluid or artificial intestinal fluid) was added at $37 \pm 0.5^{\circ} \mathrm{C}$ to compensate for the volume loss. Following filtration through a $0.8-\mu \mathrm{m}$ microporous membrane, the UV absorbance of release medium and microcapsule release medium was measured at $274 \mathrm{~nm}$.

$M S$ release from microcapsule $S R$ tablet and regular $S R$ tablet in vitro. Excipients, including HPMC (viscosity, $5 \mathrm{cPs}$ ), METHOCEL DC2 K4M (all donated by Shanghai Colorcon Coating Technology Co., Ltd., Shanghai, China) and microcrystalline cellulose (MCC; Shanghai Chineway Pharma Tech Co., Ltd., Shanghai, China) were crushed and passed through a 100-mesh sieve. Weighed MS microcapsules and excipients were uniformly mixed in a mortar, passed through a 40-mesh sieve three times and mixed with $85 \%$ ethanol to prepare a soft material. Granules were produced by passing material through a $16-$ mesh nylon screen and dried at $50-60^{\circ} \mathrm{C}$ for $1 \mathrm{~h}$. Dry granules were forced through a 16-mesh sieve and talcum powder (Shanghai Ju Qian Chemical Co., Ltd., Shanghai, China) was added, prior to being mixed uniformly and compressed.

In vitro release of MS microcapsule SR-tablets was compared with the release from conventional SR tablets (metoprolol tartrate tablets; AstraZeneca Pharmaceutical Co., Ltd.) in water. At the indicated time intervals $(0.5,2,4,6,8$, $10,12,15$ and $18 \mathrm{~h}$ ), samples of release liquid were drawn and, following filtration through a $0.8-\mu \mathrm{m}$ microporous membrane, the UV absorbance of conventional release medium and microcapsule release medium was measured at $274 \mathrm{~nm}$.

Pharmacokinetic studies of MS microcapsules in dogs. Six male beagle dogs (age, 8 months; weight, $8-10 \mathrm{~kg}$ ) were obtained from the Guangzhou General Pharmaceutical Research Institute Co., Ltd. (Guangzhou, China), housed in air-conditioned chambers at ambient temperature and humidity, fed a standard laboratory diet and a 12-h light/dark 
Table I. Levels of single factor analysis.

\begin{tabular}{|c|c|c|c|c|c|}
\hline \multirow[b]{2}{*}{ Factor } & \multicolumn{5}{|c|}{ Level } \\
\hline & 1 & 2 & 3 & 4 & 5 \\
\hline $\mathrm{W}_{1}: \mathrm{O}$ & $1: 20$ & $1: 15$ & $1: 10$ & $1: 5$ & $1: 1$ \\
\hline MS content (mg) & 1 & 2 & 3 & 4 & 5 \\
\hline EC concentration $(\mathrm{mg} / \mathrm{ml})$ & 5 & 10 & 20 & 40 & 80 \\
\hline Power of ultrasonic emulsification (W) & 40 & 80 & 120 & 160 & 200 \\
\hline Duration of ultrasonic emulsification (sec) & 30 & 60 & 90 & 120 & 180 \\
\hline PEG 6,000 concentration $(\%)$ & 1 & 2 & 3 & 4 & 5 \\
\hline $\mathrm{W}_{1} / \mathrm{O}: \mathrm{W}_{2}(\mathrm{v} / \mathrm{v})$ & $1: 5$ & $1: 10$ & $1: 15$ & $1: 20$ & $1: 25$ \\
\hline Mixing speed of multiple emulsion (rcf, $\mathrm{x} \mathrm{g}$ ) & 450 & 750 & 1,050 & 1,350 & 1,650 \\
\hline Multiple emulsion mixing time (min) & 0.5 & 1 & 2 & 3 & 4 \\
\hline Diffusion time (h) & 0.5 & 1 & 2 & 3 & 4 \\
\hline
\end{tabular}

$\mathrm{W}_{1}$, inner aqueous phase of MS; $\mathrm{O}$, organic solvent phase of EC dissolved in ethyl acetate; MS, metoprolol succinate; EC, ethyl cellulose; PEG, polyethylene glycol; $\mathrm{W}_{2}$, external aqueous phase of PEG 6,000 dissolved in ethyl acetate.

cycle. Dogs had free access to food and water. All animal experiments were performed in full compliance with local, national, ethical and regulatory principles with the approval of the Institutional Animal Care and Use Committee of China Pharmaceutical University (Nanjing, China).

Dogs were fasted overnight and randomly divided into two groups ( $\mathrm{n}=3$ in each). Each dog was orally administered $47.5 \mathrm{mg}$ MS microcapsules or $50 \mathrm{mg}$ metoprolol tartrate tablets (AstraZeneca Pharmaceutical Co., Ltd.). Prior to administration, $2 \mathrm{ml}$ venous blood was collected from the dogs, and further samples were drawn at 0.5, 1, 1.5, 2, 2.5, 3, 4, 8 and $16 \mathrm{~h}$ post-administration. Dogs had free access to food and water after $4 \mathrm{~h}$ administration. Samples were mixed with $1 \%$ sodium heparin solution anti-coagulant (Nanjing King-Friend Biochemical Pharmaceutical Co., Ltd., Nanjing, China), centrifuged for $10 \mathrm{~min}$ at $4,500 \times \mathrm{g}$, siphoned and stored at $-20^{\circ} \mathrm{C}$.

The concentration of MS in blood samples was determined using a Shimadzu 10-Avp high performance liquid chromatography system (Shimadzu, Kyoto, Japan) with a C18 column (Inertsil ${ }^{\circledR}$ ODS-SP, $4.6 \times 250 \mathrm{~mm}$; particle size, $5 \mu \mathrm{m}$, GL Sciences, Inc., Tokyo, Japan) and a mobile phase of methanol/water equal to 6/4 (v/v) (containing $960 \mathrm{mg}$ sodium heptane sulfonate and $82 \mathrm{mg}$ anhydrous sodium acetate, adjusted to $\mathrm{pH} 4.7$ with glacial acetic acid) at $30^{\circ} \mathrm{C}$; Absorbance was measured with an excitation wavelength of $285 \mathrm{~nm}$ and an emission wavelength of $316 \mathrm{~nm}$, with a sample injection volume of $50 \mu 1$.

Statistical analysis. Statistical analysis was performed with SPSS 13.0 software (SPSS Inc., Chicago, IL, USA). Values are expressed as the mean \pm standard deviation. Multiple regression was used to determine the optimal conditions for preparation of MS microcapsules. Statistical significance was determined using a two-tailed Student's t-test. Fisher's Least Significant Difference, Sidak and Tukey's post hoc analysis were applied as the post hoc analysis following one-way anal$y$ sis of variance for the homogeneity variance data. Statistical significance was set at $\mathrm{P}<0.05$.

\section{Results}

Optimization of microcapsule preparation. Microcapsules were prepared using the $\mathrm{W}_{1} / \mathrm{O} / \mathrm{W}_{2}$ double emulsification-solvent diffusion method, with $\mathrm{W}_{1}$ being an MS solution, $\mathrm{O}$ being EC dissolved in EA and $\mathrm{W}_{2}$ being PEG 6,000. The impact of the following factors on the encapsulating efficiency was analyzed: Volume ratio of $\mathrm{W}_{1}: \mathrm{O}, \mathrm{MS}$ content in $\mathrm{W}_{1}, \mathrm{EC}$ concentration, power and duration of ultrasonic emulsification, $\mathrm{PEG}$ 6,000 concentration, $\mathrm{W}_{1} / \mathrm{O}: \mathrm{W}_{2}$ volume ratio, stirring speed and time, and diffusion time (Table I).

As demonstrated in Table II, single factor analysis indicated that microcapsule loading capacity was influenced by $\mathrm{W}_{1}: \mathrm{O}$, MS content in $\mathrm{W}_{1}, \mathrm{EC}, \mathrm{W}_{1} / \mathrm{O}: \mathrm{W}_{2}$ volume ratio and multiple emulsion mixing time.

Uniform design is an algorithm used to test the influence of multiple factors with fewer experiments by evenly distributing the tested factors in the experimental design (17). Uniform design was applied to determine the optimal conditions for the preparation of microcapsules. Each factor was tested at five levels, using the principle of the intended level (repeating the same level multiple times without adding new levels), so that the number of levels reached more than twice the number of factors. A total of 15 level experiments were designed, using the $\mathrm{U}_{15}\left(15^{5}\right)$ uniform design table (18) and the resultant drug loading is illustrated in Table III.

Using mean drug loading as an index, multiple regression analysis was performed for each factor. At $\alpha=0.05$, the regression equation revealed that it was possible to calculate mean drug loading according to the formula $2.865+0.597$ $\left(\mathrm{W}_{1}: \mathrm{O}\right)+1.341\left(\mathrm{MS}\right.$ content of $\left.\mathrm{W}_{1}\right)+0.194$ (multiple emulsion mixing time $)\left(\mathrm{F}=328.265 ; \mathrm{P}<0.001 ; \mathrm{r}^{2}=0.990\right)$. Regression analysis indicated that $\mathrm{W}_{1}: \mathrm{O}$, MS content of $\mathrm{W}_{1}$ and multiple emulsion mixing time were significantly correlated with drug loading $(\mathrm{P}<0.05)$, whereas $\mathrm{EC}$ or the $\mathrm{W}_{1} / \mathrm{O}: \mathrm{W}_{2}$ volume ratio were not. The optimal microcapsule preparation conditions were determined as follows: Internal $\mathrm{W}_{1}: \mathrm{O}, 1: 1$; MS content of $\mathrm{W}_{1}, 5 \mathrm{mg}$; EC, $20 \mathrm{mg} / \mathrm{ml} ; \mathrm{W}_{1} / \mathrm{O}: \mathrm{W}_{2}$ volume ratio, 1:15; and 
Table II. Analysis of single factors affecting microcapsule loading capacity.

\begin{tabular}{lccccc}
\hline & \multicolumn{5}{c}{ Drug loading amount (\%) } \\
\cline { 2 - 5 } Factor & Level 1 & Level 2 & Level 3 & Level 4 & Level 5 \\
\hline $\mathrm{W}_{1}$ :O & $8.45 \pm 0.36$ & $8.57 \pm 0.71$ & $8.69 \pm 0.45$ & $8.73 \pm 0.38$ & $8.76 \pm 0.32$ \\
MS content & $7.14 \pm 0.28^{\mathrm{a}}$ & $7.52 \pm 0.17^{\mathrm{b}}$ & $8.68 \pm 0.31^{\mathrm{c}}$ & $9.31 \pm 0.34^{\mathrm{d}}$ & $9.72 \pm 0.51^{\mathrm{e}}$ \\
EC content & $7.89 \pm 0.21$ & $8.53 \pm 0.19$ & $8.71 \pm 0.47$ & $7.84 \pm 0.51$ & $7.16 \pm 0.15$ \\
Power of ultrasonic emulsification & $7.99 \pm 0.36$ & $8.65 \pm 0.43$ & $8.71 \pm 0.25$ & $8.91 \pm 0.78$ & $8.72 \pm 0.13$ \\
Time of ultrasonic emulsification & $8.40 \pm 0.82$ & $8.68 \pm 0.20$ & $8.69 \pm 0.27$ & $8.72 \pm 0.71$ & $8.62 \pm 0.50$ \\
Concentration of PEG 6,000 & $8.35 \pm 0.67$ & $8.39 \pm 0.58$ & $8.43 \pm 0.17$ & $8.41 \pm 0.13$ & $8.36 \pm 0.41$ \\
$\mathrm{~W}_{1} / \mathrm{O}: \mathrm{W}_{2}$ & $7.87 \pm 0.52$ & $7.91 \pm 1.16$ & $8.06 \pm 0.39$ & $8.01 \pm 0.36$ & $7.95 \pm 0.27$ \\
Mixing speed of multiple emulsion & $8.42 \pm 0.34$ & $8.67 \pm 0.28$ & $8.69 \pm 0.23$ & $8.75 \pm 0.51$ & $8.71 \pm 0.39$ \\
Multiple emulsion mixing time & $8.39 \pm 0.36^{\mathrm{f}}$ & $8.78 \pm 0.47$ & $9.02 \pm 0.68$ & $9.06 \pm 1.08$ & $9.14 \pm 0.38$ \\
Time of diffuser & $8.56 \pm 0.43$ & $8.59 \pm 0.30$ & $8.61 \pm 0.77$ & $8.58 \pm 0.50$ & $8.55 \pm 0.12$ \\
\hline
\end{tabular}

Levels are defined in Table I. Values are expressed as the mean \pm standard deviation $(n=3) . W_{1}$, inner aqueous phase of MS; O, organic solvent phase of EC dissolved in ethyl acetate; MS, metoprolol succinate; EC, ethyl cellulose; PEG, polyethylene glycol; $\mathrm{W}_{2}$, external aqueous phase of PEG 6,000 dissolved in ethyl acetate. ${ }^{\mathrm{P}} \mathrm{P}<0.05$ indicates a significant difference between level 1 and levels 3 to 5 . ${ }^{\mathrm{b}} \mathrm{P}<0.05$ indicates a significant difference between level 2 and levels 3,4 and $5 .{ }^{c} \mathrm{P}<0.05$ indicates a significant difference between level 3 and levels $1,2,4$ and $5 .{ }^{\mathrm{d}} \mathrm{P}<0.05$ indicates a significant difference between level 4 and levels 1,2 , and $3 .{ }^{~} \mathrm{P}<0.05$ indicates a significant difference between level 5 and levels 1 , 2 and 3. ${ }^{\mathrm{f}} \mathrm{P}<0.05$ indicates a significant difference between level 1 and levels 3,4 and 5 .
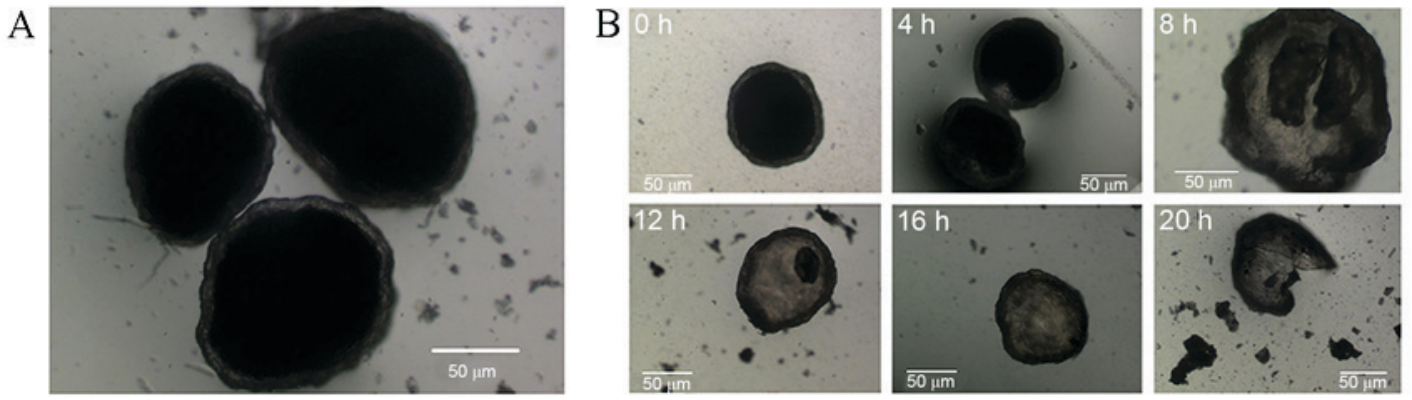

Figure 1. Microscopic images of microcapsules (magnification, x400). (A) The morphology of the microcapsules prepared was observed using bright field microscopy. (B) When re-suspended in double distilled water, the integrity of the microcapsules was maintained until rupture at $20 \mathrm{~h}$ (scale bar, $50 \mu \mathrm{m}$ ).

multiple emulsion stirring time, 4 min. The microencapsulated drug loading capacity predicted using a regression equation under optimal conditions was $10.94 \%$. Consistent with this predicted value, the three batches of microcapsules prepared were determined to have an average drug loading capacity of $10.91 \%$, as well as an encapsulation efficiency of $83.16 \%$.

Microcapsule morphology and size distribution. Prepared microcapsules were spherical, with relatively uniform size, and did not adhere to one another (Fig. 1). Microcapsule wall integrity was maintained for $20 \mathrm{~h}$ prior to rupture when suspended in distilled water, indicating a 20 -h period of diffusion-controlled drug release. Microcapsule size was relatively narrowly distributed, with $86 \%$ of capsules being 70-100 $\mu \mathrm{m}$ in diameter, and a mean particle size of $85 \mu \mathrm{m}$ (Fig. 2).

In vitro release. The in vitro drug release profiles of the MS microcapsules in various dissolution media are presented in Fig. 3. The release equations of MS microcapsules in water, artificial gastric fluid, and in artificial intestinal fluid

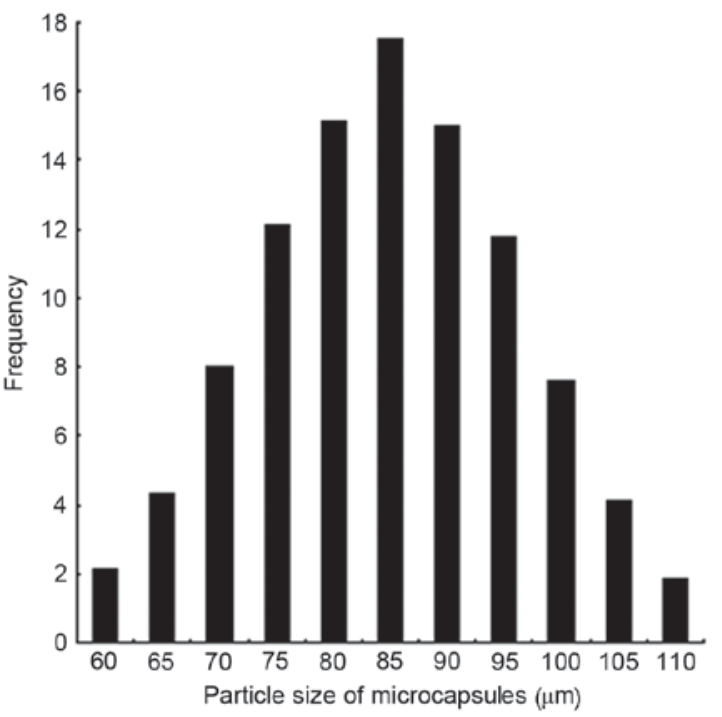

Figure 2. Particle size distribution of microcapsules. Diameters of microcapsules were measured using bright field microscopy and the frequencies of capsules with different diameters are presented. 
Table III. Assessment of the influence of multiple factors on drug loading capacity using the uniform design algorithm.

\begin{tabular}{|c|c|c|c|c|c|}
\hline $\begin{array}{l}\text { Level, } \mathrm{W}_{1}: \mathrm{O}, \\
(\mathrm{v} / \mathrm{v})\end{array}$ & $\begin{array}{c}\text { Level, MS } \\
\text { content (mg) }\end{array}$ & $\begin{array}{c}\text { Level, EC } \\
\text { concentration (mg/ml) }\end{array}$ & $\begin{array}{c}\text { Level, } \\
\mathrm{W}_{1} / \mathrm{O}: \mathrm{W}_{2}(\mathrm{v} / \mathrm{v})\end{array}$ & $\begin{array}{l}\text { Level, multiple emulsion } \\
\text { mixing time (min) }\end{array}$ & $\begin{array}{c}\text { Drug } \\
\text { loading }(\%)\end{array}$ \\
\hline $1(1: 20)$ & $4(2)$ & $7(20)$ & $11(1: 20)$ & $13(4)$ & $6.27 \pm 1.46$ \\
\hline $2(1: 20)$ & $8(3)$ & $14(80)$ & $7(1: 15)$ & $11(3)$ & $7.18 \pm 1.39$ \\
\hline $3(1: 20)$ & $12(4)$ & $6(10)$ & $3(1: 5)$ & $9(2)$ & $8.61 \pm 1.08$ \\
\hline $4(1: 15)$ & $1(1)$ & $13(80)$ & $14(1: 25)$ & $7(2)$ & $4.31 \pm 0.73$ \\
\hline $5(1: 15)$ & $5(2)$ & $5(10)$ & $10(1: 20)$ & $5(1)$ & $5.74 \pm 0.97$ \\
\hline $6(1: 15)$ & $9(3)$ & $12(40)$ & $6(1: 10)$ & $3(0.5)$ & $6.86 \pm 1.33$ \\
\hline $7(1: 10)$ & $13(5)$ & $4(10)$ & $2(1: 5)$ & $1(0.5)$ & $9.69 \pm 1.55$ \\
\hline $8(1: 10)$ & $2(1)$ & $11(40)$ & $13(1: 25)$ & $14(4)$ & $4.88 \pm 0.89$ \\
\hline $9(1: 10)$ & $6(2)$ & $3(5)$ & $9(1: 15)$ & $12(3)$ & $6.17 \pm 1.29$ \\
\hline $10(1: 5)$ & $10(4)$ & $10(40)$ & $5(1: 10)$ & $10(3)$ & $8.77 \pm 1.76$ \\
\hline $11(1: 5)$ & $14(5)$ & $2(5)$ & $1(1: 5)$ & $8(2)$ & $10.06 \pm 2.21$ \\
\hline $12(1: 5)$ & $3(1)$ & $9(20)$ & $12(1: 20)$ & $6(1)$ & $4.45 \pm 0.62$ \\
\hline $13(1: 1)$ & $7(3)$ & $1(5)$ & $8(1: 15)$ & $4(1)$ & $7.66 \pm 1.92$ \\
\hline $14(1: 1)$ & $11(4)$ & $8(20)$ & $4(1: 10)$ & $2(0.5)$ & $8.84 \pm 1.57$ \\
\hline $15(1: 1)$ & $15(5)$ & $15(80)$ & $15(1: 25)$ & $15(4)$ & $10.63 \pm 1.86$ \\
\hline
\end{tabular}

Each factor was tested at five levels using the principle of the intended level (repetition of the same level multiple times without addition of new levels), so that the number of levels reached more than twice the number of factors. A total of 15 level experiments were designed, using the $\mathrm{U}_{15}\left(15^{5}\right)$ uniform design table. Values are expressed as the mean \pm standard deviation $(n=3)$. $\mathrm{W}_{1}$, inner aqueous phase of MS; O, organic solvent phase of EC dissolved in ethyl acetate; MS, metoprolol succinate; EC, ethyl cellulose; PEG, polyethylene glycol; $\mathrm{W}_{2}$, external aqueous phase of PEG 6,000 dissolved in ethyl acetate.

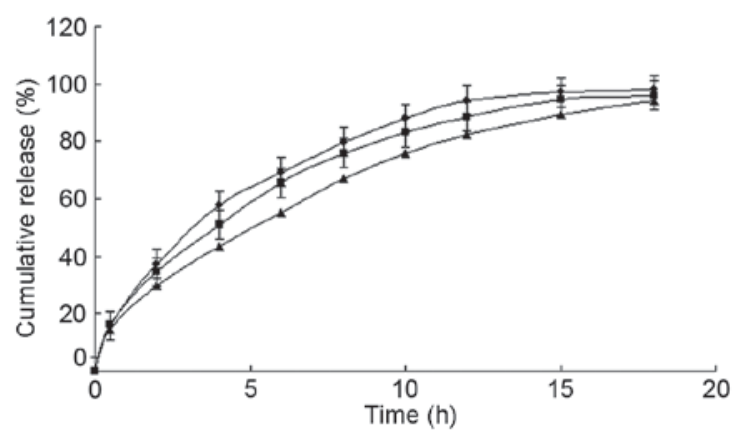

Figure 3. Drug release from MS microcapsules in vitro. Prepared microcapsules were placed in a basket with $1,000 \mathrm{ml}$ of release medium (water, artificial gastric fluid or artificial intestinal fluid). Samples were taken from the release solution at pre-determined time intervals $(0.5,2,4,6,8,10,12$, 15 and $18 \mathrm{~h}$ ) to detect the released drug concentration. The total percentages of MS released at different time-points are plotted. Values are expressed as the mean \pm standard deviation $(n=12)$. $\mathbf{n}$, water; $\bullet$, artificial gastric fluid; $\boldsymbol{\Delta}$, artificial intestinal fluid. MS, metoprolol succinate.

were determined to be $\mathrm{Q}=22.346 \mathrm{t}^{1 / 2}+1.6068(\mathrm{r}=0.992)$; $\mathrm{Q}=26.438 \mathrm{t}^{1 / 2}+2.5376(\mathrm{r}=0.990)$; and $\mathrm{Q}=22.553 \mathrm{t}^{1 / 2}-1.3337$ $(\mathrm{r}=0.997)$, respectively. The cumulative release was found to fit the Higuchi equation (19), indicating that MS was released from microcapsules via diffusion. The MS microcapsules demonstrated a good SR profile in all three media, with a mean release of $96.1 \%$ within $18 \mathrm{~h}$.

When submerged in water, conventional SR-tablets exhibited a burst of release with $>50 \%$ released in $2 \mathrm{~h}$. Conversely, the MS microcapsule SR-tablets exhibited an almost linear SR in a 12-h test period (Fig. 4).
Pharmacokinetic studies in vivo. Pharmacokinetic studies of MS microcapsules and conventional SR tablets were performed in dogs to evaluate the in vivo performance of these formulations (Fig. 5). The results indicated that conventional tablets and microcapsules fitted a single-compartment model. The pharmacokinetic parameters are presented in Table IV. Both formulations exhibited a similar area under curve; however, the microcapsules exhibited a significantly longer half-life and time to peak, and a markedly lower maximum drug concentration $\left(\mathrm{C}_{\max }\right)$.

\section{Discussion}

SR formulations of MS may minimize fluctuations in plasma concentration, avoiding the adverse effects associated with excessively high plasma concentrations and providing a stable effective dose. Microspheres have been previously used in SR formulations of MS to provide robust and consistent control of hypertension and heart rate (20). The objective of the present study was to develop a method of MS encapsulation to provide microcapsules with high entrapment efficiency and optimal sustained-release profiles in vitro and in vivo.

EC was employed as the capsule material and EA as a solvent to prepare multiple emulsions. At room temperature, the emulsion was added to distilled water to cause phase separation and condensation of microcapsules. The solvent was removed with excess water, avoiding the elevated temperatures usually employed in solvent evaporation methods, which may have negatively affected the stability of the encapsulated compound. Water-insoluble EC was employed as a blocker film coating. 
Table IV. Pharmacokinetic parameters.

\begin{tabular}{lcc}
\hline Parameter & $\begin{array}{c}\text { Conventional } \\
\text { SR-tablets }\end{array}$ & $\begin{array}{c}\text { SR } \\
\text { microcapsules }\end{array}$ \\
\hline $\mathrm{t}_{1 / 2}(\mathrm{~h})$ & $0.84 \pm 0.09$ & $2.84 \pm 0.37^{\mathrm{a}}$ \\
$\mathrm{Ka}(1 / \mathrm{h})$ & $0.88 \pm 0.12$ & $0.255 \pm 0.10^{\mathrm{a}}$ \\
$\mathrm{T}_{\max }(\mathrm{h})$ & $1.17 \pm 0.27$ & $4.01 \pm 0.53^{\mathrm{a}}$ \\
$\mathrm{C}_{\max }(\mathrm{ng} / \mathrm{ml})$ & $216.13 \pm 48.79$ & $86.69 \pm 27.71^{\mathrm{a}}$ \\
$\mathrm{CL}(\mathrm{l} / \mathrm{kg})$ & $0.42 \pm 0.07$ & $0.43 \pm 0.09$ \\
$\mathrm{AUC}(\mathrm{ng} \cdot \mathrm{h} / \mathrm{ml})$ & $738.50 \pm 150.82$ & $710.71 \pm 131.64$ \\
\hline
\end{tabular}

Values are expressed as the mean \pm standard deviation $(n=3) . t_{1 / 2}$, tablet half life; $\mathrm{Ka}$, absorption rate; $\mathrm{T}_{\max }$, time to peak drug concentration; $\mathrm{C}_{\max }$, maximum drug concentration; $\mathrm{CL}$, drug clearance; $\mathrm{AUC}$, area under curve. ${ }^{\mathrm{a}} \mathrm{P}<0.05$ vs. control.

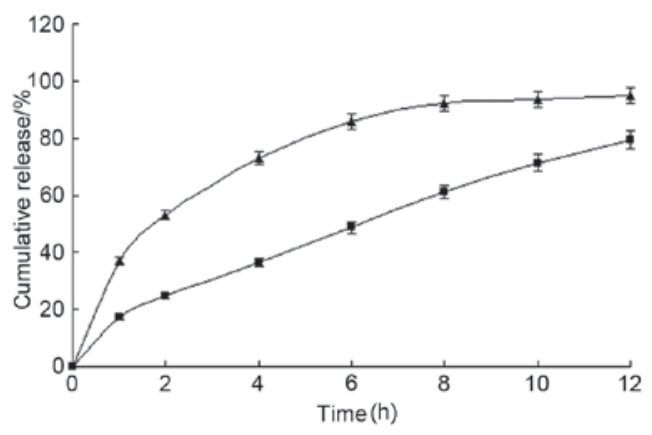

Figure 4. Drug release from metoprolol succinate microcapsule SR tablets and conventional SR tablets in water were detected and release profiles compared. Data are presented as the mean \pm standard deviation $(n=12)$. $\mathbf{n}$, MS microcapsules SR tablets; $\boldsymbol{\Delta}$, MS conventional SR tablets. MS, metoprolol succinate; SR, sustained-release.

Increased ultrasonic power and time during emulsification may increase drug loading; however, excessive emulsification may lead to excess evaporation of EA in the primary emulsion. In the current study, when agitated too quickly, excessive foam was produced and the primary emulsion particle size decreased, reducing the dose loaded per particle. Emulsification at $<160 \mathrm{~W}$ with a mixing time of $<120 \mathrm{sec}$ and $1,350 \times \mathrm{g}$ using PEG 6,000 as a plasticizer in the $\mathrm{W}_{2}$ phase produced microcapsules with favorable mechanical properties, as walls were less prone to rupture and drug leakage was reduced. An excess of PEG may reduce the amount of drug loading and accelerate drug release, and may also cause microcapsules to aggregate into larger clumps, with oil leach induced by mechanical impact adversely affecting the process of preparation (21). As such, the optimal PEG 6,000 concentration was determined to be $3 \%$. It was also necessary to determine the optimum diffusion time, as excessively long diffusion periods may result in loss of the pharmacological agent (20). The optimum diffusion time was determined to be $2 \mathrm{~h}$.

Given the complexity of the microcapsule preparation process, numerous variables may be adjusted to optimize the drug loading capacity. By using a single factor method and applying a uniform design to assess the contribution of

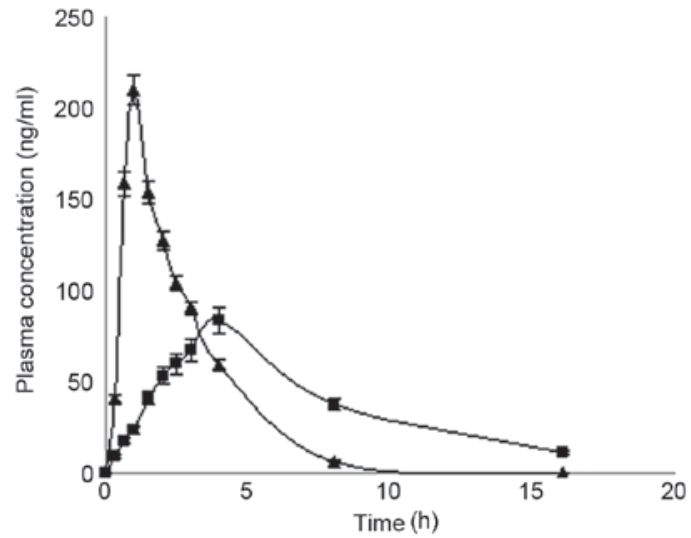

Figure 5. Microcapsule drug release profile in vivo. Six dogs were fasted overnight and administered $47.5 \mathrm{mg}$ MS microcapsules or $50 \mathrm{mg}$ metoprolol tartrate tablets (conventional SR tablets). Venous blood samples harvested prior to and following administration at $0.5,1,1.5,2,2.5,3,4,8$ and $16 \mathrm{~h}$ were used to detect MS concentration by HPLC analysis. Values are expressed as the mean \pm standard deviation $(n=3)$. $\mathbf{m}$, SR microcapsules; $\boldsymbol{\Delta}$, conventional SR tablets. MS, metoprolol succinate; SR, sustained-release; HPLC, high-performance liquid chromatography.

individual parameters, and subsequently performing multiple regression analysis to select the optimal conditions, the loading capacity of MS microcapsules was optimized (22). The MS microcapsules prepared under optimal conditions exhibited good morphological characteristics, a high encapsulation efficiency and good reproducibility.

It is difficult to achieve prolonged SR of water-soluble pharmacological agents with conventional tablets (23). Microcapsules formed with EC and PEG 6,000 exhibited SR of MS in vivo and in vitro. The 18-h release behavior followed the Higuchi equation in water (19), and release rates in artificial gastric and intestinal fluid were similar and reproducible, with a release of $\sim 80 \%$ of the encapsulated MS within $12 \mathrm{~h}$. Furthermore, the novel microcapsule formula developed in the present study exhibited slower in vitro release of MS than the conventional tablet formula. These novel microcapsules are advantageous, as they achieve a more even SR in various environments, including water, artificial gastric fluid and artificial intestinal fluid.

When dogs were orally administered microcapsules and conventional tablets, the plasma half-life of MS with the microcapsule tablet formulation was longer than with the conventional tablet and the peak plasma concentration was significantly lower $(\mathrm{P}<0.05)$. MS microcapsule SR tablets may provide additional advantages by reducing the dose frequency and minimizing adverse effects associated with $\mathrm{C}_{\max }$.

In conclusion, the present study reported on the development of microcapsules with high entrapment efficiency and desirable SR properties in vitro and in vivo. An entrapment efficiency of $83.16 \%$ was achieved and $96.1 \%$ of MS was released in vitro within $18 \mathrm{~h}$. Pharmacokinetic studies of MS microcapsules in dogs indicated a superior SR profile compared with conventional SR tablets. These findings suggested that the use of microcapsules in tablets may provide therapeutic benefits over conventional tablets by SR of pharmacological agents. 


\section{Acknowledgements}

The authors would like to thank Professor Liu at Guangzhou General Pharmaceutical Research Institute for providing the animal models used in the present study.

\section{References}

1. Papadopoulos DP and Papademetriou V: Metoprolol succinate combination in the treatment of hypertension. Angiology 60: 608-613, 2009.

2. Reiter MJ: Cardiovascular drug class specificity: Beta-blockers. Prog Cardiovasc Dis 47: 11-33, 2004.

3. Frishman WH and Saunders E: $\beta$-Adrenergic blockers. J Clin Hypertens (Greenwich) 13: 649-653, 2011.

4. Bengtsson C, Johnsson $\mathrm{G}$ and Regårdh CG: Plasma levels and effects of metoprolol on blood pressure and heart rate in hypertensive patients after an acute dose and between two doses during long-term treatment. Clin Pharmacol Ther 17: 400-408, 1975.

5. Guan G and Li Z: Evaluation of the effect on metoprolol succinate sustained release tablets in reducing blood pressure. Jian Yan Yi Xue Yu Lin Chuang 6: 1989-1990, 2009 (In Chinese)

6. Chirra HD and Desai TA: Emerging microtechnologies for the development of oral drug delivery devices. Adv Drug Deliv Rev 64: 1569-1578, 2012.

7. Lu B, Wen R, Yang H and He Y: Sustained-release tablets of indomethacin-loaded microcapsules: Preparation, in vitro and in vivo characterization. Int J Pharm 333: 87-94, 2007.

8. Frishman WH, Hainer JW and Sugg J; M-FACT Study Group: A factorial study of combination hypertension treatment with metoprolol succinate extended release and felodipine extended release results of the Metoprolol Succinate-Felodipine Antihypertension Combination Trial (M-FACT). Am J Hypertens 19: 388-395, 2006.

9. Siddique S, Khanam J and Bigoniya P: Development of sustained release capsules containing 'coated matrix granules of metoprolol tartrate'. AAPS PharmSciTech 11: 1306-1314, 2010.

10. Gong X, Lu Y, Xiang Z and Luo G: Preparation of polysulfone microcapsules containing 1-octanol for the recovery of caprolactam. J Microencapsul 26: 104-110, 2009.

11. Zhang X, Zhu Y, Wang J, Sun Y, Li S and Pan W: Preparation of sustained-release microspheres of clarithromycin by quasi-emulsion solvent diffusion method. Zhongguo Yao Ye 18: 39-40, 2009 (In Chinese).
12. You J, Cui FD, Han X, Wang YS, Yang L, Yu YW and Li QP: Study of the preparation of sustained-release microspheres containing zedoary turmeric oil by the emulsion-solvent-diffusion method and evaluation of the self-emulsification and bioavailability of the oil. Colloids Surf B Biointerfaces 48: 35-41, 2006.

13. Jelvehgari M and Montazam SH: Comparison of microencapsulation by emulsion-solvent extraction/evaporation technique using derivatives cellulose and acrylate-methacrylate copolymer as carriers. Jundishapur J Nat Pharm Prod 7: 144-152, 2012.

14. Dash V, Mishra SK, Singh M, Goyal AK and Rath G: Release kinetic studies of aspirin microcapsules from ethyl cellulose, cellulose acetate phthalate and their mixtures by emulsion solvent evaporation method. Sci Pharm 78: 93-101, 2010.

15. Saringat HB, Alfadol KI and Khan GM: The influence of different plasticizers on some physical and mechanical properties of hydroxypropyl methylcellulose free films. Pak J Pharm Sci 18: 25-38, 2005 .

16. Huang G, Deng S, Wang $\mathrm{R}$ and $\mathrm{Xi}$ Y: Preparation and in vitro-in vivo correlation in dogs of metoprolol succinate sustained-released tablets. Zhongguo Yi Yao Gong Ye Za Zhi 36: 412-414, 2005 (In Chinese).

17. $\mathrm{Xu} \mathrm{W}, \mathrm{Li} \mathrm{N}$ and Gao C: Preparation of controlled porosity osmotic pump tablets for salvianolic acid an doptimization of the formulation using an artificial neural network method. Acta Pharm Sin B 1: 64-70, 2011.

18. Shen LN, Zhang YT, Wang Q, Xu L and Feng NP: Enhanced in vitro and in vivo skin deposition of apigenin delivered using ethosomes. Int J Pharm 460: 280-288, 2014

19. Saurí J, Millán D, Suñé-Negre JM, Colom H, Ticó JR, Miñarro M, Pérez-Lozano P and García-Montoya E: Quality by design approach to understand the physicochemical phenomena involved in controlled release of captopril SR matrix tablets. Int J Pharm 477: 431-441, 2014

20. Albin P, Markus A, Ben-Zvi Z and Pelah Z: A new slow release formulation of metoprolol: In-vitro and in-vivo evaluation in dogs. J Control Release 23: 1-11, 1993.

21. Liu Y, Feng Y and Xu D: Influence of excipients on the mechanical properties of microcapsules. Chin Traditional Pat Med 29: 1602-1605, 2007

22. Zhao W: Uniform design application in pharmaceutics. Anhui Med Pharm J 14: 610-612, 2010.

23. Pradhan R, Kim YI, Chang SW and Kim JO: Preparation and evaluation of once-daily sustained-release coated tablets of tolterodine-L-tartrate. Int J Pharm 460: 205-211, 2014. 